\title{
El Problema de la Obesidad y sus Efectos Sobre el Mercado Laboral Chileno
}

\author{
Cristian Raggio Carvallo* \\ UNIVERSIDAD DE CHILE
}

\section{RESUMEN}

El actual problema de la obesidad en Chile trae consecuencias desfavorables en términos sociales y económicos, tales como elevados gastos de salud en que debe incurrir el Estado y penalidades en los resultados laborales para individuos que presentan esta condición, hacia lo cual se ha inclinado la evidencia empírica. En este sentido, considerando que a la fecha de este estudio más de un $25 \%$ de la población chilena es obesa, se hace urgente estimar las potenciales pérdidas que esta realidad implica sobre la economía del país. Por ende, haciendo uso del panel de la encuesta de protección social (EPS), proponemos una serie de especificaciones de efectos fijos para analizar los efectos de la obesidad sobre productividad, participación y probabilidad de empleo, a la vez que revisaremos la relación entre esta condición y diferentes medidas de salud, en un intento por reconocer los canales a través de los cuales altos índices de masa corporal (IMC) afectan el mercado laboral. Así, fuimos capaces de demostrar un efecto negativo no lineal en las mujeres para todas las medidas que estudiamos, apreciando tanto un efecto independiente de la obesidad (el cual puede ser asociado a una discriminación laboral), como uno indirecto reflejado por el peor estado de salud que implica tener un alto IMC.

Palabras claves: Obesidad, Índice de masa corporal (IMC), Efectos Fijos, Discriminación.

\section{Abstract}

The problem of obesity in Chile brings adverse consequences in social and economic terms, such as higher health costs for the State and penalties on labor outcomes for people that have this condition, to where the empiric evidence has inclined. So, if we consider that at the date of this study more than $25 \%$ of chilean population is obese, it becomes urgent to estimate the potential losses that this reality implies over the country's economy. Therefore, using panel data from the "Encuesta de Protección Social" (EPS), we propose fixed effects especifications to analize the effects of obesity over productivity, participation and employment, while also looking at the relation between this condition and different health measures, in an effort to recognize the channels through which high body mass indexes (BMI) affects the labor market. We were able to demonstrate a negative and non linear effect for women among all the labor measures that we study, observing both an independent effect of obesity (that could be asociated with discrimination) and one indirect reflected by the worse health condition as a result of a high BMI.

Keywords: Obesity, Body Mass Index (BMI), Fixed Effects, Discrimination.

*craggio@fen.uchile.cl 


\section{INTRODUCCIÓN}

asta hace algunas décadas, uno de los problemas sociales
predominantes en el mundo era la desnutrición y sus graves
efectos sobre la salud y calidad de vida de la población. La mortalidad infantil era elevada, mientras que el crecimiento y productividad de los Estados se veía mermado por los problemas de salud derivados de la mala alimentación (Strauss \& Thomas, 1998). Es así como fueron surgiendo modelos de salarios de eficiencia en la búsqueda de una relación entre nutrición y productividad de los trabajadores. Leibenstein (1957), en uno de los trabajos precursores de esta corriente estableció que, relativo a trabajadores malnutridos, aquellos que tienen una dieta más rica en calorías son más productivos, relación que resultó ser no lineal para niveles de consumo calórico bajos, donde una mejor alimentación se asocia a incrementos considerablemente mayores en productividad.

En los últimos veinte a treinta años sin embargo, ha tenido lugar una rápida transición del problema mencionado anteriormente hacia el de la obesidad, tanto en el mundo desarrollado como en países en vías de desarrollo (Uauy, Albala and Kain, 2001) (por supuesto, la desnutrición sigue estando presente en los países económicamente más atrasados). En este contexto, la mortalidad infantil por falta de alimentos pasó a ser reemplazada por crecientes índices de sobrepeso en los menores, así como una reducción de la esperanza de vida y aumento de los costos sociales y económicos derivados de esta condición ${ }^{1}$. A grandes rasgos, estos costos podemos dividirlos en dos: (1) El gasto en salud pública que el Estado debe realizar para hacer frente a este problema y a las enfermedades que se ven potencialmente desarrolladas por los malos hábitos alimenticios, a la vez que se ha argumentado acerca del (2) costo que implica para la productividad, y como consecuencia sobre la economía, una fuerza de trabajo con altos niveles de sobrepeso y obesidad.

Con respecto al primer punto, la asociación entre la obesidad y enfermedades no transmisibles tales como la diabetes, hipertensión, cardiovasculares y diversos tipos de cáncer ha sido ampliamente corroborada en la literatura de salud ${ }^{2}$. Por otro lado, existen 
numerosos estudios que discuten el efecto de la obesidad sobre resultados laborales tales como salarios y o participación laboral, donde la evidencia empírica es más bien mixta ( $D^{\prime}$ Hombres \& Brunello, 2005; Morris, 2006; Sargent \& Blanchflower, 1994; Sousa, 2005). Lo anterior tiene varias explicaciones que van desde el contexto económico y socio cultural de un país o región, hasta lo meramente econométrico (diferentes estrategias empíricas utilizadas y limitaciones en cuanto a datos). Dado esto, se hace fundamental realizar cuidadosamente estas estimaciones, en un tiempo y espacio determinado, de forma tal que los resultados se acerquen lo más posible a la realidad de cada caso. Así, el primer objetivo del presente trabajo será estimar el efecto de la obesidad sobre la productividad y participación, aprovechando la coyuntura para analizar también los efectos sobre la probabilidad de empleo (y así dar luces sobre una potencial discriminación laboral), en un país en vías de desarrollo como lo es Chile.

Para abordar esta pregunta de investigación, debemos tener en cuenta que a pesar que en la literatura médica solo ha sido demostrado el efecto negativo de la obesidad (y no sobrepeso o incrementos del IMC en el tiempo de personas con peso normal) sobre la salud (SBU, 2002), algunos trabajos han encontrado una relación causal negativa entre el IMC como variable continua y resultados laborales (Sargent \& Blanchflower, 1994; Baum \& Ford, 2004; d'Hombres $\&$ Brunello, 2005). Por lo tanto, podríamos observar la presencia de otros factores distintos a los de salud que explican la correlación existente entre altos niveles de masa corporal y el mercado del trabajo, tales como discriminación por género/belleza, peor estado físico de los individuos o mayores tasas de impaciencia, entre otros. Luego, el siguiente objetivo del paper es establecer un link entre obesidad y diferentes medidas de la salud de los individuos, para contrastar estos resultados con los obtenidos a nivel laboral y ver si efectivamente ese es el canal a través del cual se reflejan los efectos en productividad, participación y probabilidad de empleo. 


\section{REVISIÓN LITERATURA}

Existe una larga y creciente literatura que busca determinar las consecuencias de la obesidad en el mercado laboral. Esta condición puede mostrar una correlación negativa con salarios por varias razones, dentro de las cuales podemos mencionar la discriminación (ya sea por parte de los empleadores y/o consumidores), además de características personales tales como "flojera" o falta de habilidades sociales, lo cual ha sido asociado recurrentemente a los obesos (Sobal, 2004) y que al final de cuentas determinan una menor productividad. Por otro lado, los obesos tienden a mostrar mayores tasas de impaciencia con respecto al futuro, haciéndolos más propensos a descuidar su alimentación y a su vez invertir menos en capital humano (Zhan and Rashad, 2008; Cawley, 2004).

Como contraparte, el hecho de mostrar dificultades en sus actividades interpersonales implica al mismo tiempo una limitante para establecer una relación de pareja (Gortmaker et. al, 1993). Luego, al creer que sus posibilidades de matrimonio son bajas, personas con un alto IMC pueden realizar una mayor inversión en capital humano, consiguiendo mejores salarios (Averett and Korenman, 1996). Finalmente, la cultura de cada país puede jugar un rol importante a la hora de penalizar o no esta condición en el mercado laboral (Costa-Font and Gil, 2004; García and QuintanaDomeque, 2006).

Baum \& Ford (2004) estiman varias especificaciones diferentes de salarios, incluyendo un modelo de efectos fijos para controlar el potencial sesgo por heterogeneidad no observable. Para hacer esto, incluyen una serie de características exógenas (background) del individuo, a la vez que comparan cambios en los salarios a través del tiempo y entre hermanos (aprovechando un extenso panel de datos). Este último modelo se basa en el supuesto que las diferencias de no observables entre hermanos eliminan la variación del peso atribuible al ambiente familiar compartido, incluyendo en parte el factor genético. Ahora bien, es importante destacar que la literatura sobre la genética detrás de los comportamientos personales no ha sido capaz de encontrar un efecto del ambiente familiar compartido 
sobre el peso (Murphy \& Topel, 1985). Prosiguiendo, los autores examinan una serie de canales a través de los cuales la obesidad puede afectar salarios. Para realizar esto, testean si los trabajadores obesos ganan menos porque i) están limitados por restricciones de salud, ii) son más miopes en términos económicos (factores internos), iii) son más costosos para los empleadores que proveen cuidados de salud, o bien iv) son discriminados por los clientes (factores externos).

Otra alternativa de estimación es el método de variables instrumentales, el cual es preferido al de efectos fijos si creemos que los factores no observables sí varían a través del tiempo. En este sentido, surge en la literatura la idea de utilizar una medida rezagada de la variable IMC ya que tiene una menor probabilidad de reflejar causalidad inversa, al limpiar el efecto contemporáneo del salario sobre el nivel de masa corporal del individuo. Luego, en un esfuerzo por darle mayor robustez a estos modelos, Averett \& Korenman (1996) juntaron ambas metodologías reemplazando el peso actual por su rezago y controlando por efectos fijos entre hermanos utilizando datos de corte transversal, mientras que Conley \& Glauber (2005) tomaron rezagos del IMC (13 y 15 ańos atrás) como instrumentos para el actual índice y también estimaron un modelo de efectos fijos entre hermanos. Ambos estudios encontraron efectos negativos de la obesidad sobre productividad, sin embargo, a pesar de que estos modelos son capaces de limpiar factores no observables permanentes a nivel familiar, los efectos individuales que pueden estar afectando obesidad y salarios al mismo tiempo se mantienen en el término de error.

Siguiendo esta alternativa para abordar la endogeneidad de estos modelos, existe un enfoque metodológico que intenta tomar en cuenta los no observables que pueden ser adjudicados a la genética del individuo, para lo cual se ha utilizado el IMC de un miembro familiar biológico como instrumento. Es el caso del trabajo realizado por Cawley (2000), el cual utiliza el peso de los hijos como instrumento del peso corporal de sus madres sin encontrar efectos negativos de la obesidad sobre sector ocupacional y participación laboral, mientras que en salarios, solo se encontró evidencia de una 
penalidad para mujeres de raza blanca (sus pares de habla hispana mostraron una débil correlación y las de raza negra ninguna).

Norton \& Han (2008) por su parte, argumentan que información genética de genes específicos que han sido relacionados con la obesidad en la literatura biomédica proveen un instrumento ideal para la obesidad ya que los genes son una fuente de variación exógena. Demuestran que esta información genética en conjunto con el IMC del hermano predice de buena forma el rezago del IMC del individuo y su probabilidad de tener sobrepeso. Sin embargo, Baum \& Ford (2004) se muestran escépticos ante esta metodología explicando que los modelos de efectos fijos son una mejor aproximación al problema de endogeneidad en estos estudios, ya que la técnica de variables instrumentales se basa en asumir restricciones de exclusión y sobre el supuesto que las variables excluidas no están correlacionadas con los efectos fijos no observables. En este último trabajo, al igual que el realizado por Han, Norton \& Stearns (2008) se controla por los efectos fijos individuales, pero no utilizan variables instrumentales por ser débiles (poca variación exógena), y en el mejor de los casos, solamente identificadas.

Finalmente, cabe mencionar el trabajo por Pagan \& Davila (1997) donde utilizan en primera instancia un logit multinomial para obtener información acerca de la selección ocupacional de individuos con sobrepeso y luego estiman ecuaciones de salarios a nivel transversal, tomando en cuenta el logro ocupacional de estos últimos. Sus resultados encuentran una penalidad en salarios para las mujeres, mientras que los hombres al tener mayores facilidades para moverse entre sectores ocupacionales (autoselección), son capaces de evitar este efecto negativo sobre sus ingresos.

Como podemos ver, los resultados encontrados en la literatura son variados para las medidas revisadas del mercado laboral (productividad y participación) presumiblemente por las diferentes estrategias empíricas utilizadas para cada estudio, en conjunto con la variación de factores difíciles de encontrar en los datos, tales como el contexto sociocultural de cada región geográfica para la cual se hicieron las estimaciones. Esta última dificultad puede 
ser simplemente consecuencia de efectos distintos para diferentes regiones, sin embargo el problema de simultaneidad entre salud y resultados laborales es más delicado y por ende lo abordaremos en la siguiente sección. De hecho algunos trabajos de este tipo han evitado la estimación que relaciona obesidad con participación laboral debido a la fuerte simultaneidad del caso (García \& QuintanaDomeque, 2006). En este último estudio, los autores proponen estimar la relación entre masa corporal y el status laboral del individuo (empleado, trabajador por cuenta propia, desempleado) a través de un logit multinomial ya que esta especificación tiene la ventaja de proveer señales acerca de la existencia de una potencial discriminación hacia los obesos.

\section{MARCo ECONOMÉTRICO}

El problema de identificación más común en este tipo de estudios es el de doble causalidad, que surge cuando las mismas medidas no observables que determinan o están correlacionadas con el estado físico del individuo simultáneamente estén afectando salarios/ participación (heterogeneidad no observable). En otras palabras, un mejor salario permite al individuo llevar a cabo una mayor inversión en su salud (alimentación, actividad física), lo cual a su vez se ve reflejado en más productividad laboral. Luego, no es posible identificar una relación causal entre obesidad y este tipo de variables si no se controla por los factores no observables, resultando en una estimación sesgada.

Para tratar este problema, los datos de panel son de gran utilidad ya que muestran diferentes observaciones en el tiempo para un mismo individuo, lo que permite la estimación de modelos de efectos fijos individuales. Bajo esta especificación, el término de error se descompone en dos: un componente fijo que representa la heterogeneidad no observable específica a cada individuo de la muestra (la cual asumimos que no varía en el tiempo), y otro aleatorio que cumple con los supuestos MCO. De esta forma la ecuación genérica toma la siguiente expresión:

$$
Y_{i t}=\alpha_{i}+\beta X_{i t}+u_{i t}
$$


Donde $\mathrm{Y}$ es la variable dependiente, $\mathrm{X}$ es un vector de variables explicativas que incluye medidas del IMC, $\alpha_{i}$ es el componente específico ya definido y $u$ el error aleatorio que se asume iid con media cero y varianza constante. Luego, como la información contenida $\bar{\kappa}_{i}$ no es observable para el investigador e incluye características personales tales como la motivación o hábitos alimenticios, y a la vez creemos que este tipo de características correlacionan simultáneamente con salarios e IMC, entonces los parámetros estimados se encontraran sesgados. Ante esta situación el método de efectos fijos toma la diferencia en el tiempo de al menos dos observaciones para cada entrevistado, eliminando así las variables no observables que se encuentran afectando la estimación, siempre bajo el supuesto que estas no fluctúan de un período al otro, es decir, $\alpha_{i t}=\alpha_{i t}-1$.

$$
Y_{i t}-Y_{i t-1}=\left(\alpha_{i t}-\alpha_{i t-1}\right)+\beta\left(X_{i t}-X_{i t-1}\right)+\left(u_{i t}-u_{i t-1}\right)
$$

Un atributo adicional de esta técnica es su capacidad para reducir el sesgo de selección siempre presente en las ecuaciones de salarios. Este sesgo aparece porque los individuos que se encuentran trabajando decidieron previamente participar o no en el mercado laboral dado una serie de factores personales, es decir, se autoseleccionan en un grupo particular y por ende los resultados de la estimación (que solo utiliza observaciones donde salario $>0$ ) no serán válidos para toda la fuerza de trabajo. En nuestro caso, el sesgo de selección se controla si asumimos que cualquier factor no observable que determine el empleo se mantiene fijo en el tiempo (se incluye dentro de $\alpha_{i}$ ) y por lo tanto es eliminado al estimar por primeras diferencias.

Con todo, después de revisar la literatura y entendiendo las ventajas que proporcionan este tipo de modelo, las especificaciones a estimar consideraran siempre el efecto fijo individual utilizando el método de primeras diferencias, tanto para el caso de regresión múltiple $\mathrm{MCO}$ con variable dependiente continua (salarios y salud), como para los modelos de probabilidad lineal (MPL) con variable dependiente binaria (participación, enfermedad y empleo).

Para estos tres últimos casos, se escogió el MPL por sobre la estimación de máxima verosimilitud (MV) dado que tanto en el modelo Logit como el Probit no es posible controlar por efectos 
fijos individuales ya que la no linealidad de sus funciones no permite suprimir $\kappa_{i}$ al tomar las primeras diferencias. La alternativa entonces es estimar por MV pero bajo efectos aleatorios. En esta modalidad, el componente propio a cada individuo es obtenido de una distribución con media cero y varianza constante (va incluido en el término de error) y por ende presenta autocorrelación en el tiempo. Además, se asume exogeneidad de los regresores con el componente individual, es decir, que $\operatorname{cov}\left(\alpha_{i}, X\right)=0$. Pero como ya fue expuesto, existe una gran cantidad de no observables que afectan tanto el IMC del individuo como sus resultados laborales y por lo tanto si aplicamos efectos aleatorios obtendríamos un estimador considerablemente sesgado ${ }^{3}$.

A pesar de que el MPL es la alternativa más viable dado nuestro contexto muestral, debemos también constatar las falencias de este enfoque, que no impone ninguna restricción para que el rango de las probabilidad predicha se encuentre entre cero y uno, lo que implica que debemos ser muy cuidadosos con la interpretación de los resultados porque al asumir que una probabilidad correlaciona de manera lineal con una variable explicativa continua (escolaridad, por ejemplo), aumentos constantes en esta pueden llevar la probabilidad estimada fuera del intervalo $[0,1]$. Otro potencial problema es que el error no se distribuye de forma normal, lo que va de la mano con una varianza de los residuos heterocedástica. La mejor manera de lidiar con esta limitante es estimando errores estándar robustos a la heterocedasticidad ${ }^{4}$. En efecto, como cada individuo provee más de una observación que no son independientes, se debe controlar por la correlación entre estas ya que si existe, los errores estándar estarán subestimados mientras que los niveles de significancia se encontrarán sobreestimados.

Finalmente, con respecto a la elección de nuestra variable de interés, existe en la literatura una interesante discusión acerca de cuál es la mejor medida para evaluar los efectos de la obesidad sobre los resultados laborales, surgiendo dos alternativas predominantes. Una es la construcción de variables dicotómicas que toman el valor de 1 para cada rango del IMC, la cual tiene la capacidad de 
capturar posibles efectos no lineales; hipótesis plausible si creemos por ejemplo que la penalidad en salarios es mayor para individuos obesos mórbidos que para sus pares con sobrepeso. Además, esta especificación nos permite estimar el efecto por separado de cada clasificación y compararlos. La otra opción es incluir el IMC como variable continua y de esta forma estimar cómo cambia nuestra variable dependiente ante una variación marginal en la masa corporal de las personas. A su vez, para capturar no linealidades se puede utilizar un polinomio (potencias) de esta variable.

Para efectos de este estudio, nos enfocaremos en la primera alternativa, ya que nos permite obtener conclusiones más detalladas sobre la respuesta del mercado del trabajo ante las diferentes caracterizaciones de peso de los individuos y además es más directa a la hora de interpretar los resultados de las estimaciones.

Concluimos esta sección presentando las ecuaciones que dictan relación con el mercado del trabajo y en seguida las de salud.

$\underline{\text { Salarios }}$

$$
W_{i t}=\alpha_{i}+\beta_{1} X_{i t}+\beta_{2} A c t_{i t}+\beta_{3} D i s_{i t}+\beta_{4} E_{i t}+\beta_{5} D I M C_{i t}+u_{i t}
$$

Con W como el logaritmo natural del salario por hora (del individuo i en el tiempo t); X un conjunto de controles de capital humano; Act contienen controles por actividad económica; Dis toma valor 1 si el individuo posee algún tipo de discapacidad física; E toma valor 1 si el individuo padece una de las siguientes enfermedades: asma/efisema, depresión, diabetes, hipertensión, problemas cardíacos, y finalmente DIMC como un vector de dummies para cada rango del $\mathrm{IMC}^{5}$, donde se omite en la estimación el rango normal para evitar la colinealidad y además poder hacer la inferencia con respecto a este grupo.

Participación

$$
P_{i t}=\alpha_{i}+\beta_{1} X_{i t}+\beta_{2} Z_{i t}+\beta_{3} D i s_{i t}+\beta_{3} E_{i t}+\beta_{4} D I M C_{i t}+u_{i t}
$$

Donde $\mathrm{P}$ toma valor 1 si la persona participa del mercado laboral ${ }^{6}$ y cero si se encuentra inactivo; los controles $\mathrm{X}$, Dis, E y las dummies DIMC ya fueron definidas, y se agrega en $\mathrm{Z}$ el estado civil (=1 si está casado) y el número de hijos del individuo. Ambos factores son utilizados generalmente en este tipo de estimaciones ya que influyen 
en la decisión de participación, sobre todo en el caso de las mujeres dado el contexto cultural de Chile.

Empleo

$$
T_{i t}=\alpha_{i}+\beta_{I} X_{i t}+\beta_{2} Z_{i t}+\beta_{3} D i s_{i t}+\beta_{3} E_{i t}+\beta_{4} D I M C_{i t}+u_{i t}
$$

Con $\mathrm{T}=1$ si la persona se encuentra trabajando y cero si no. Por su parte las variables que explican esta condición son las mismas incluidas en la ecuación de participación.

$\underline{\text { Salud }}$

$$
H_{i t}=\alpha_{i}+\beta_{I} X_{i t}+\beta_{2} D i s_{i t}+\beta_{3} E_{i t}+\beta_{4} F_{i t}+\beta_{5} D I M C_{i t}+u_{i t}
$$

Donde $\mathrm{H}$ es un auto reporte del estado de salud hecho por cada individuo $^{7}$; X, Dis y E ya fueron definidas, y se agrega $\mathrm{F}$ que toma valor 1 si el individuo fuma tabaco. Por último están las dummies IMC.

\section{$\underline{\text { Enfermedad }}$}

$$
E_{i t}=\alpha_{i}+\beta_{i} X_{i t}+\beta_{2} D i s_{i t}+\beta_{3} F_{i t}+\beta_{4} D I M C_{i t}+u_{i t}
$$

Donde E toma valor 1 si el individuo posee alguna(s) de las 5 enfermedades ya mencionadas, y los demás controles al igual que nuestra variable de interés ya son conocidos.

\section{DAtos}

Para examinar los efectos de la obesidad en la salud, salarios y participación laboral por género se utilizó la Encuesta de Protección Social (EPS), la cual es de carácter longitudinal tipo panel, con una muestra de alrededor de 16.000 encuestados distribuidos en todas las regiones del país, y hasta la actualidad se han realizado cinco rondas $(2002,2004,2006,2009$ y 2012). En la encuesta se recolecta información acerca de la historia laboral y características personales de cada individuo, mientras que las preguntas del ámbito de salud (que incluyen peso y estatura) fueron agregadas recién en la segunda ronda de la encuesta (2004). Además, cabe destacar que la última ronda no se considera un producto logrado y por ende no es posible utilizarla con fines de inferencia estadística, por el nivel de error que esto puede conllevar. De esta forma, tenemos un panel que abarca tres rondas y una diferencia de 5 años entre la primera y la última.

Claramente, lo ideal hubiese sido contar con todas las rondas de 
la encuesta y así asegurar una variación considerable en las variables a incluir en los modelos, además de tener una mayor cantidad de observaciones en la muestra, sin embargo esto no fue posible y como consecuencia existirá una limitación en cuanto a los datos, ya que es difícil encontrar cambios importantes en las variables que nos interesa medir en un período tan corto de tiempo (pensemos por ejemplo en peso, estatura y salarios).

Con todo, tenemos una muestra total inicial de 18.778 individuos cuya edad va desde los 16 ańos en adelante. Sin embargo, las observaciones en las cuales los hombres midan menos de 140 centímetros y las mujeres reporten una estatura menor a 135 centímetros serán removidas de la muestra ya que son considerados como outliers, en el sentido que esos rangos de estatura se escapan de lo normal y posiblemente tengan alguna enfermedad de crecimiento, lo que puede sesgar nuestros resultados si no controlamos por estos no observables (también pueden corresponder a errores de medición). Sumado al ajuste anterior, no son considerados en las estimaciones los entrevistados que trabajan en las fuerzas armadas o en trabajos por cuenta propia ${ }^{8}$, ya que en estos casos los individuos no se encuentran propensos a sufrir una discriminación/penalización en el mercado laboral (pero si serán considerados para la estadística descriptiva). Después de limpiar la muestra nos quedan 17.780 individuos y un total de 41.434 observaciones, de las cuales 21.950 son mujeres $(52,98 \%)$ y 19.484 hombres.

\section{IV.1 Contexto de la Obesidad en Chile}

Antes de presentar la estadística descriptiva de los datos, voy a discutir brevemente el contexto de la obesidad en Chile, así como su evolución en los últimos años. Lo anterior con el objeto de sentar un precedente acerca de la magnitud del problema que estamos tratando de abordar en este estudio, además de permitirnos ponerlo en perspectiva previo a la revisión de los datos.

Existen varios factores que explican la situación nutricional de los chilenos en la actualidad, dentro de los que podemos encontrar cambios en los hábitos alimenticios, disminución en la práctica 
de actividades físicas, una gradual caída en la tasa de natalidad y mejoras en términos socioeconómicos. Todo esto en las últimas dos a tres décadas (Atalah, 2011).

Los cambios demográficos como el envejecimiento poblacional, sumado a una transición cultural hacia la constitución de familias más pequeñas con mayor espacio entre generaciones, han permitido a los padres proveer de una mejor alimentación a sus hijos (Vio, Albala \& Kain, 2008). Adicionalmente, el crecimiento económico en este período, a pesar de no hacerse cargo de las desigualdades en el país, ha sido fundamental para disminuir la pobreza, lo que se ve traducido en una mayor capacidad de consumo para las familias de menores recursos, aunque esto no necesariamente implica el acceso a alimentos más saludables y es aquí donde comienza el problema en cuestión. La comida consumida dentro y fuera del hogar es de un alto contenido calórico, una realidad inconsistente con el nivel de sedentarismo que existe en nuestro país, sobre todo para los niveles socioeconómicos más bajos.

Según cifras de la Encuesta Nacional de Salud', para el año 2003 un $89,4 \%$ de la población total era sedentaria durante su tiempo libre, situación que se mantiene para la segunda ronda de la encuesta en el año 2010 (88,6\%), donde las mujeres sobresalen con 92.9\% de sedentarismo. Luego, al dividir esta misma muestra por nivel educacional, vemos una caída en la proporción de sedentarios a medida que aumenta la escolaridad $(96,9 \%$ para el nivel bajo, $88,6 \%$ para el medio y $82,2 \%$ para el alto).

Con respecto a la población adulta, que es de especial interés para efectos de nuestro estudio, los índices de obesidad y obesidad mórbida aumentaron drásticamente en el período entre encuestas ( 21,9 a $25,1 \%$ y 1,2 a $2,3 \%$, respectivamente) mientras que los individuos con sobrepeso alcanzaron el 39,3\% de la población adulta en 2010. Sumando estos tres porcentajes llegamos a que, a esa fecha, un $63,5 \%$ de los adultos chilenos tenía un IMC por sobre lo considerado normal. Cabe destacar que estas variaciones son de suma importancia si buscamos identificar los efectos de la obesidad en modelos de diferencias, considerando que el espacio entre ambas 
encuestas de salud es similar al período para el cual poseemos datos. IV.2 Estadística Descriptiva.

Ahora a partir del contexto país descrito y luego de presentar la muestra a utilizar en este trabajo, comenzaremos la descripción de los datos con un análisis de la evolución de la obesidad en la fuerza laboral, separando la muestra por género y entregando valores promedios para salarios y participación (tablas 1 y 2 de anexos). Para realizar esto, primero debemos definir nuestra variable IMC que es el ratio entre el peso y la estatura del individuo al cuadrado. De esta forma, según la clasificación para adultos propuesta por la OMS, tenemos:

TABLA 1: RANGOS IMC.

\begin{tabular}{|c|c|}
\hline Clasificación & IMC $(\mathbf{k g} / \mathbf{m} \mathbf{2})$ \\
\hline Bajo Peso & $<18,5$ \\
\hline Normal & {$[18,5 ; 25)$} \\
\hline Sobrepeso & {$[25 ; 30)$} \\
\hline Obeso & {$[30 ; 40)$} \\
\hline Obeso Mórbido & $\geq 40$ \\
\hline
\end{tabular}

Fuente: OMS, clasificación IMC.

Disponible: apps.who.int/bmi/index.jsp?introPage=intro_3.html

Si apreciamos la tabla 1 (anexos) correspondiente a la muestra completa, vemos como ha aumentado el porcentaje de individuos con sobrepeso, obesidad y obesidad mórbida, para hombres y mujeres. Lo contrario ocurre con los otros dos rangos (normal y bajo peso), donde existe un gradual descenso de estos índices a través de los años. Así, aunque las magnitudes del aumento son un poco menores a la información entregada por la ENS (lo cual se puede explicar porque nuestra muestra no incluye individuos menores a 16 años y como sabemos la obesidad infantil en Chile es considerable), podemos corroborar la tendencia de la población hacia peores niveles de peso/salud. Finalmente, si sumamos los porcentajes del total de individuos con IMC por sobre el normal para el año 2009, llegamos a una cifra muy similar al 63,5\% que obtuvo la ENS.

Ahora para referirnos a la evolución de salarios y participación nos centraremos únicamente en la muestra para cual realizaremos 
nuestras estimaciones, es decir, la fuerza laboral del país (personas de 15 ańos y más, que se encuentran en la situación de ocupados o desocupados) sin contar los trabajadores por cuenta propia ni FFAA (tabla 2, anexos). A priori, lo que podemos observar es que en cada una de las rondas, los hombres con sobrepeso y obesos en promedio ganan más y tienen una mayor participación laboral que sus pares con IMC normal (lo cual resulta contradictorio con la evidencia internacional). Incluso los hombres obesos mórbidos muestran un salario más alto que aquellos con rango de peso normal en las tres encuestas. Los ingresos y participación de las mujeres en cambio sí siguen un comportamiento a la baja según aumenta el rango de peso, lo que puede argumentarse de varias formas entre las que se encuentra una potencial discriminación de género-belleza, o también a través de la hipótesis propuesta por Pagan \& Davila (1997) sobre una mayor facilidad de los hombres obesos para moverse entre sectores ocupacionales y así no recibir una penalización en el salario por su condición física. Por último, cabe destacar que el aumento del salario a través de los años para todos los grupos (menos las mujeres con IMC mayor a cuarenta) refleja principalmente el hecho de que esta medida está en términos nominales y por ende no es posible obtener conclusiones acerca de una variación en el poder de compra (salario real) de las personas.

En conclusión, no es posible distinguir una correlación negativa entre las medidas del mercado laboral y el IMC para los hombres, mientras que para las mujeres es evidente que existe. Dado esto, haremos estimaciones para toda la muestra y separando por género, considerando que la trayectoria de salarios y participación es muy diferente en cada caso. Luego, para determinar el efecto causal de la obesidad es necesario controlar por todos aquellos factores que pueden estar correlacionados con el estado físico del individuo y afectando salarios/participación a la vez, por lo que proponemos un conjunto de variables explicativas ampliamente utilizadas en la estimación de este tipo de modelos, omitiendo por supuesto los factores que no varían en el tiempo. En las siguientes tablas se presenta una estadística descriptiva de estos controles, relacionados 


\section{con el mercado laboral y la salud respectivamente.}

\section{Tabla 2: Estadística Descriptiva Mercado Laboral.}

\begin{tabular}{lcccccc} 
& \multicolumn{5}{c}{ IMC<30 } & IMC $>=30$ \\
\hline Variables dependientes & & & & & & \\
\hline Log Salario/Hora & 8,483 & $(0,716)$ & 8,488 & $(0,712)$ & 8,456 & $(0,739)$ \\
Participación & 0,531 & $(0,498)$ & 0,545 & $(0,497)$ & 0,465 & $(0,498)$ \\
\hline Variables explicativas & & & & & & \\
\hline IMC & 26,357 & $(4,375)$ & 24,884 & $(2,825)$ & 33,398 & $(3,545)$ \\
Edad & 47,469 & $(16,163)$ & 47,024 & $(16,489)$ & 49,595 & $(14,316)$ \\
Escolaridad & 9,71 & $(4,433)$ & 9,893 & $(4,446)$ & 8,837 & $(4,264)$ \\
Sexo (Hombre=1) & 0,47 & $(0,499)$ & 0,487 & $(0,499)$ & 0,388 & $(0,487)$ \\
E. Civil (Casado=1) & 0,51 & $(0,499)$ & 0,497 & $(0,5)$ & 0,573 & $(0,494)$ \\
Hijos & 1,476 & $(1,28)$ & 1,434 & $(1,275)$ & 1,678 & $(1,286)$ \\
Agricultura & 0,056 & $(0,23)$ & 0,058 & $(0,235)$ & 0,045 & $(0,208)$ \\
Minería & 0,009 & $(0,095)$ & 0,009 & $(0,096)$ & 0,007 & $(0,085)$ \\
Manufactura & 0,065 & $(0,246)$ & 0,067 & $(0,251)$ & 0,051 & $(0,221)$ \\
Electricidad agua y gas & 0,003 & $(0,062)$ & 0,004 & $(0,063)$ & 0,003 & $(0,058)$ \\
Construcción & 0,05 & $(0,219)$ & 0,052 & $(0,222)$ & 0,044 & $(0,205)$ \\
Comercio y Hotelería & 0,09 & $(0,287)$ & 0,093 & $(0,291)$ & 0,077 & $(0,268)$ \\
Transporte & 0,04 & $(0,197)$ & 0,039 & $(0,195)$ & 0,046 & $(0,209)$ \\
Financiero & 0,043 & $(0,203)$ & 0,046 & $(0,209)$ & 0,029 & $(0,168)$ \\
Servicios sociales & 0,163 & $(0,369)$ & 0,164 & $(0,37)$ & 0,155 & $(0,362)$ \\
Actividades no especificadas & 0,008 & $(0,09)$ & 0,009 & $(0,094)$ & 0,004 & $(0,067)$ \\
\hline Prnedios mesc
\end{tabular}

Promedios muestrales y desviaciones estándar (en paréntesis) para una muestra total de 41.434 observaciones de las cuales 34.267 no son obesos y 7.167 sí lo son.

Tabla 3: Estadística Descriptiva Salud.

\begin{tabular}{lcccccc} 
& & & IMC $<30$ & IMC $>=30$ \\
\hline Variables dependientes & & & & & & \\
\hline Salud & 3,272 & $(0,972)$ & 3,226 & $(0,97)$ & 3,493 & $(0,951)$ \\
Enfermedad & 0,323 & $(0,467)$ & 0,293 & $(0,455)$ & 0,465 & $(0,498)$ \\
\hline $\begin{array}{l}\text { Variables explicativas } \\
\text { (dummies) }\end{array}$ & & & & & & \\
\hline Discapacitado & 0,092 & $(0,289)$ & 0,087 & $(0,283)$ & 0,112 & $(0,315)$ \\
Fuma & 0,313 & $(0,464)$ & 0,324 & $(0,468)$ & 0,261 & $(0,439)$ \\
Asma/Efisema & 0,04 & $(0,198)$ & 0,036 & $(0,188)$ & 0,06 & $(0,238)$ \\
Depresión & 0,089 & $(0,284)$ & 0,082 & $(0,275)$ & 0,12 & $(0,325)$ \\
Diabetes & 0,069 & $(0,254)$ & 0,055 & $(0,229)$ & 0,134 & $(0,341)$ \\
Hipertensión & 0,211 & $(0,408)$ & 0,184 & $(0,387)$ & 0,341 & $(0,474)$ \\
Problemas cardíacos & 0,057 & $(0,233)$ & 0,053 & $(0,224)$ & 0,079 & $(0,27)$ \\
\hline Promios
\end{tabular}

Promedios muestrales y desviaciones estándar (en paréntesis) para una muestra total de 41.434 observaciones de las cuales 34.267 no son obesos y 7.167 sí lo son. 
Podemos ver que casi no hay diferencias en salarios, pero si hay una menor participación de los individuos obesos. Además, estos últimos tienden a educarse menos, contraer matrimonio más a menudo y son preferentemente mujeres. Lo anterior guarda relación con las teorías del comportamiento de los obesos expuestas en la revisión literaria, en cuanto a que la mayor tasa de impaciencia de estos individuos los puede llevar a invertir menos en capital humano, versus el desarrollo de peores habilidades interpersonales (y por ende, menor probabilidad de casarse) y una mayor inversión en educación. El análisis concluye con las actividades económicas, donde es difícil identificar una autoselección de los obesos en algún trabajo en particular, dada la poca diferencia en proporción con sus pares no obesos en todos los sectores revisados.

Siguiendo con la descripción de los datos, el auto reporte del estado de salud refleja una percepción levemente más negativa en los obesos, mientras que la dummy enfermedad, construida a partir de las últimas cinco variables explicativas de esta tabla $(=1$ si el individuos presenta alguna de estas condiciones), claramente se presenta más en personas con un IMC alto y lo anterior se confirma una vez que revisamos la media de las cinco variables por separado. Acá es importante recordar que así como algunas variables de salud son incluidas como controles en las ecuaciones de participación y productividad, las variables de capital humano (edad y educación) también son utilizadas para controlar el efecto de la obesidad sobre las medidas de salud de los individuos ${ }^{10}$.

\section{Resultados}

El análisis de los efectos de la obesidad sobre salarios comienza estimando el modelo (1), que incluye como controles las variables explicativas provenientes de la tabla 2. Sin embargo, es posible que el estado de salud de los individuos tenga un efecto sobre la productividad de estos, por lo tanto, para evaluar la robustez de nuestros resultados ante cambios en la especificación del modelo y a la vez separar el efecto de IMC de factores de salud, se incluyen gradualmente dos controles (enfermedad y discapacidad). 
Tabla 4: Salarios.

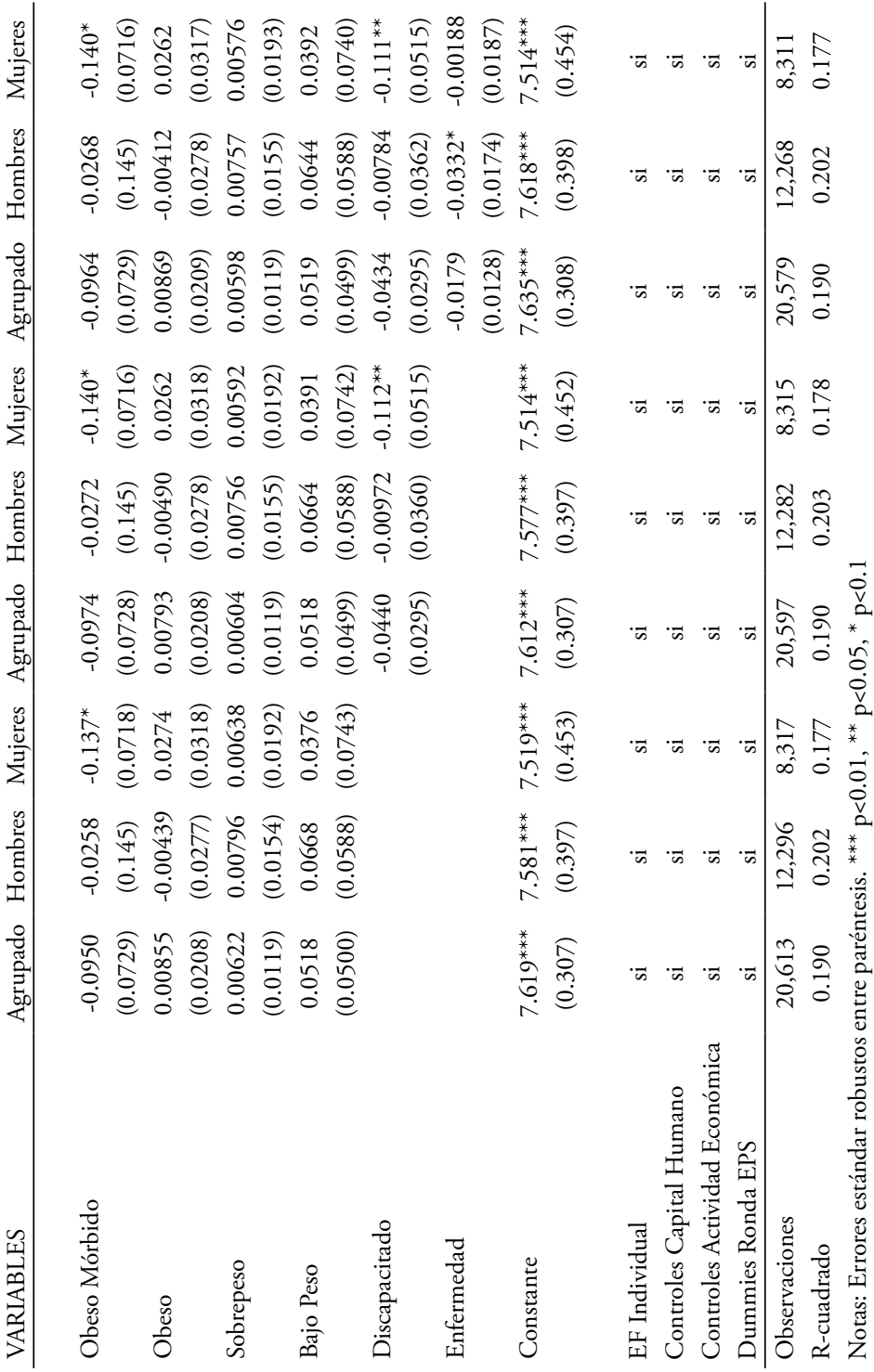

En primera instancia, solo las mujeres obesas mórbidas reciben una penalización en su salario, equivalente a un $13,7 \%$ de su renta. 
Luego, al ir agregando controles de salud, vemos que este resultado se mantiene e incluso aumenta a un $14 \%$ con un p-value de 0.051 , lo que es un buen indicio de que nuestras estimaciones no se encuentran sesgadas. Además, con esto podemos inferir que parte del efecto de la obesidad en salarios es independiente de la salud y discapacidad, ya que al controlar por estas variables el efecto no disminuye. De esta forma se abre la posibilidad de que la penalidad para las mujeres provenga de una discriminación laboral.

Los resultados para los hombres en cambio carecen de significancia estadística en todas las especificaciones y por ende no es posible demostrar un efecto de su masa corporal sobre productividad.

Es importante aquí traer a la discusión el sesgo de selección que podría estar afectando las estimaciones de las mujeres principalmente, dado que su tasa de participación es bastante menor a la de los hombres. Como fue explicado en el marco econométrico, enfrentamos esta limitante a través del uso del efecto fijo, el cual se encarga de eliminar los no observables que pueden estar afectando la decisión de participación una vez que se toman las primeras diferencias. Luego, aunque no estamos seguros de controlar todo el sesgo, al menos podemos acotarlo, asumiendo que la mayoría de las variables omitidas que afectan la decisión (pensemos por un momento en características personales, psicológicas), no varían en el tiempo.

Lo siguiente es estimar como afecta la obesidad en la decisión de participación de las personas, y siguiendo el mismo formato que el análisis de salarios, agregamos paulatinamente variables de salud que posiblemente afectan esta decisión. 
Tabla 5: Participación.

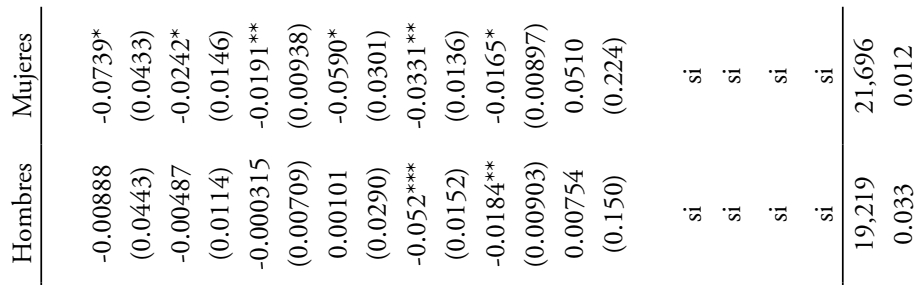

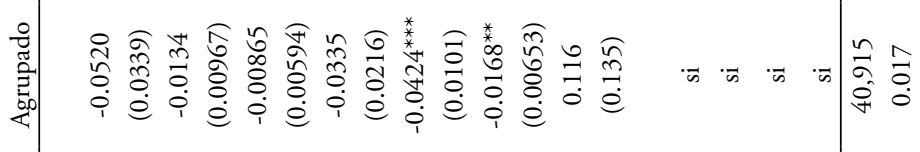

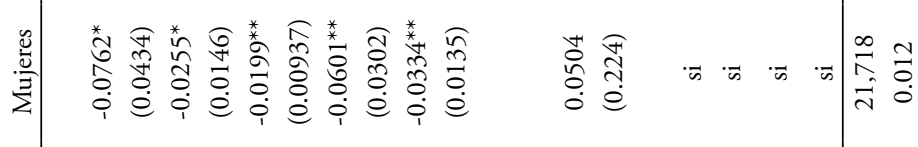

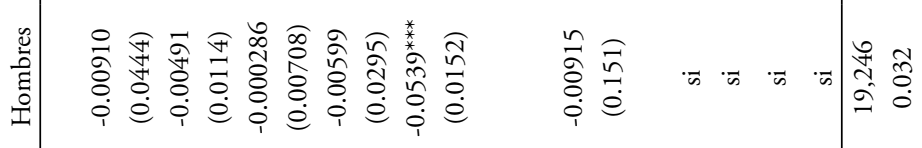

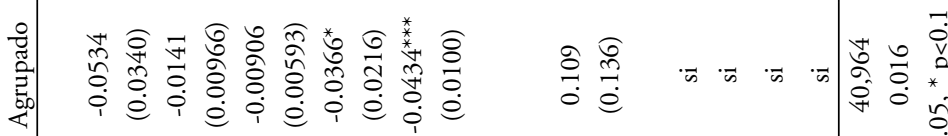

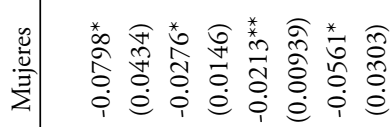

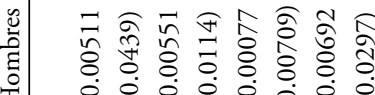

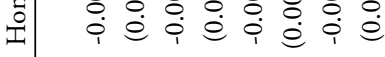

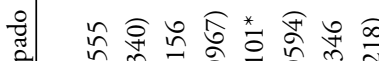

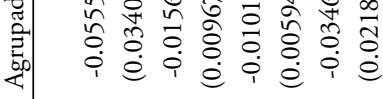

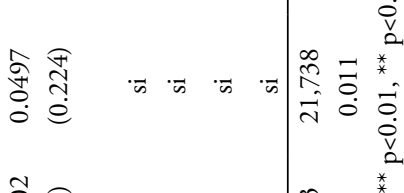

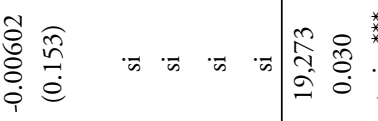

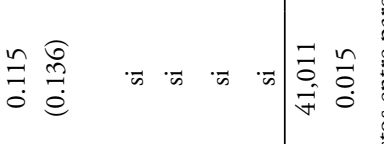

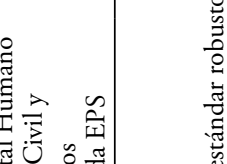

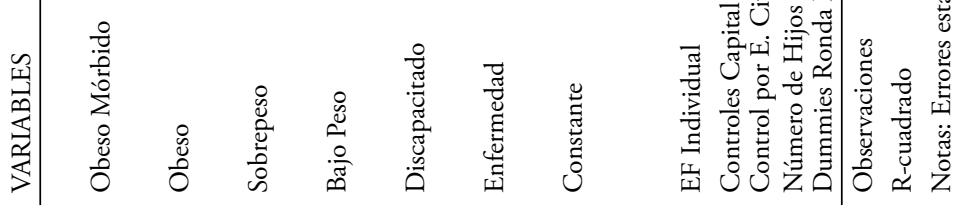


En la primera especificación para toda la muestra, solo los individuos con sobrepeso muestran una menor probabilidad de participar en el mercado del trabajo, sin embargo, al separar la muestra por sexo, las mujeres muestran un efecto negativo en todos los rangos fuera del normal, claramente con diferentes magnitudes en sus coeficientes, siendo las con un IMC por sobre 40 las con menor probabilidad de participación $(-7,98 \%)$. Por su parte, los coeficientes de obesidad no tienen significancia estadística en el caso de los hombres, lo cual se mantiene en las tres especificaciones y era predecible después de revisar la estadística descriptiva de la sección anterior.

Los controles de salud afectan considerablemente la decisión de participar y por ende, al no considerarlos estaríamos sobreestimando el efecto de los rangos de IMC. Esta es la razón por la cual las magnitudes de los estimadores van decreciendo a medida que se agregan las variables de discapacidad y enfermedad, aunque estos siguen siendo significativos y no presentan grandes cambios, lo que asegura robustez a nuestra estimación. Con esto, una vez más estamos en presencia de un efecto de la obesidad independiente del estado de salud del individuo y la explicación más plausible sigue siendo una potencial discriminación hacia las mujeres con altos niveles de masa corporal.

Por último, al estimar los modelos (1) y (2) con la variable continua del IMC y sus potencias (anexos, tabla 3 y 4), y después de escoger la especificación por criterios de información, no encontramos efecto alguno en el mercado laboral tanto en hombres como para las mujeres. Esto puede ser consecuencia de que los resultados encontrados anteriormente se concentran en personas con obesidad mórbida, por lo que al incluir el IMC de forma lineal, e incluso con polinomios, no permite identificar un efecto en salarios/ participación.

Hasta acá nuestros resultados muestran que el efecto de la obesidad es negativo para las mujeres incluso después de controlar por su estado de salud. Sin embargo, y tal como lo seńala la literatura médica, la obesidad también tiene un efecto directo sobre la salud 
de los individuos y por lo tanto podría estar afectando tanto salarios como participación indirectamente a través de la salud. Es por esto que, a continuación, analizamos los efectos de la obesidad sobre variables de salud de los individuos. Ahora bien, antes de proseguir con la inferencia, es necesario constatar nuevamente que nuestra metodología no nos permite reconocer exactamente cuáles son los canales a través de los cuales la obesidad refleja las perdidas en productividad y participación (ni menos en qué proporción afecta cada cual), sin embargo, es posible saber si la salud efectivamente es uno de estos, y luego dependiendo de qué tan grande en magnitud es la correlación con las medidas del IMC, podremos poner al descubierto la urgencia de este problema en ambos rubros (obesidadsalud y obesidad-salud-trabajo). 
TABla 6: SAlud.

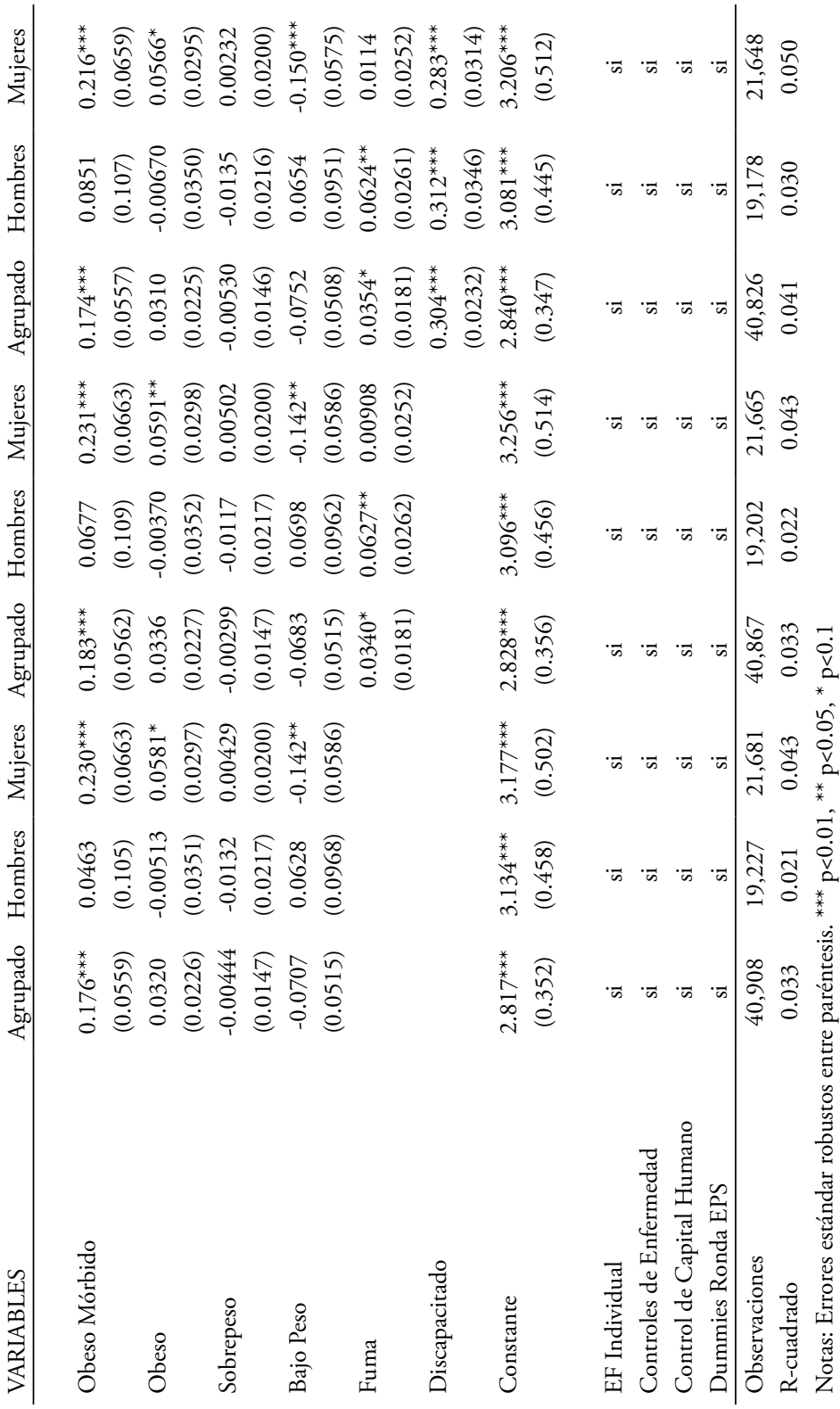


Recordemos que en el modelo (4) la variable dependiente es un auto reporte de salud que va desde excelente a muy mala y es probable que posea error de medida, sin embargo, como la variable se encuentra al lado izquierdo de la ecuación, este error se acumula en el residuo y por lo tanto no sesga los estimadores de la regresión. Por su parte, la variable enfermedad es de carácter binaria y lo que se estima en (5) es la probabilidad de contraer alguna de las enfermedades definidas en la estadística descriptiva ${ }^{11}$. 
Tabla 7: Enfermedad.

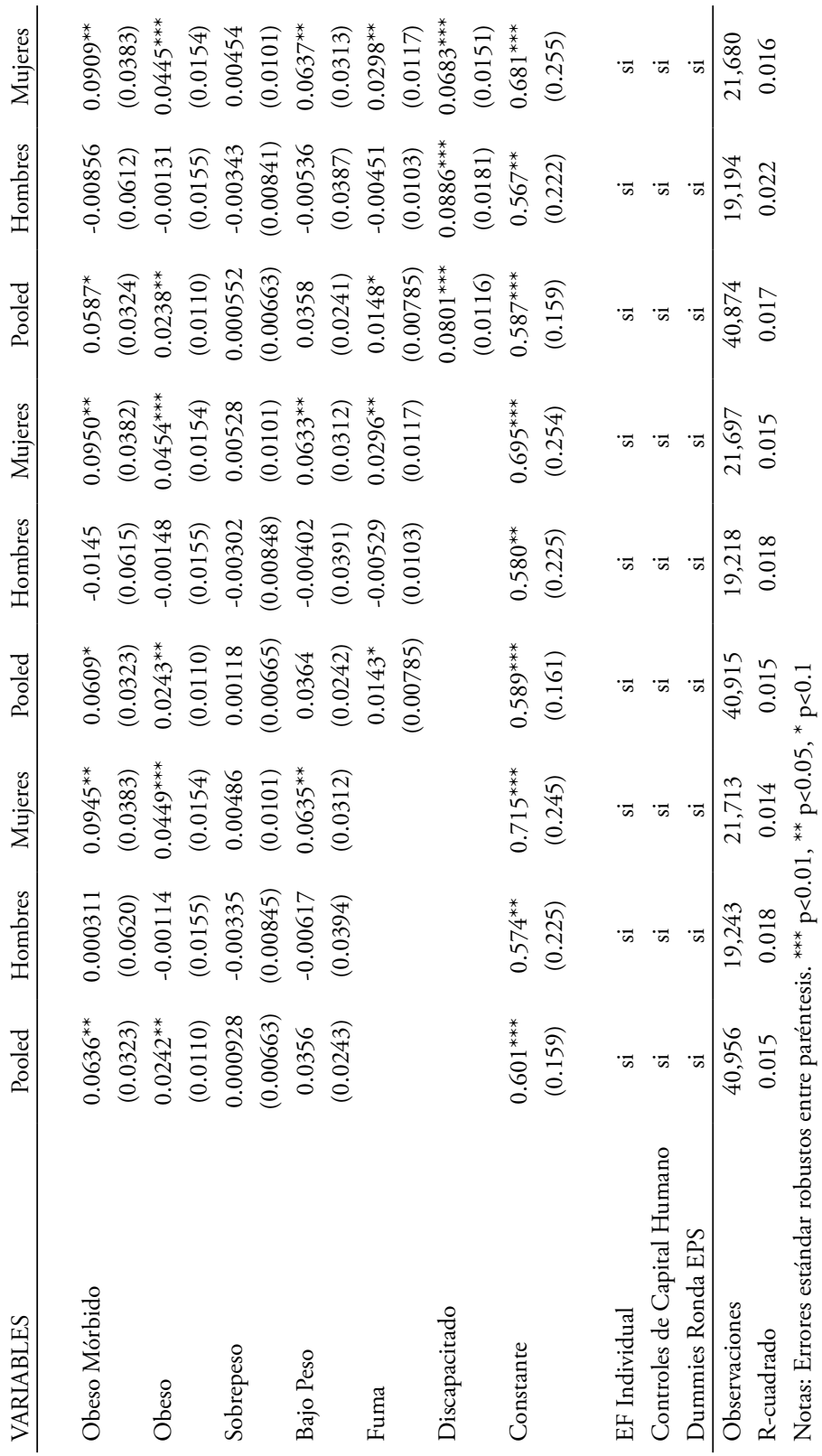


El análisis indica que los resultados obtenidos en (4) y (5) van en línea con lo encontrado anteriormente, en el sentido que una de las razones por las cuales los hombres no sufren penalidades en el mercado laboral es que su condición física no se ve reflejada en un peor estado de salud, mientras que los menores niveles de salarios y participación observado en las mujeres a medida que aumenta su IMC sobre el rango normal sí puede ser explicado en parte por los problemas de salud asociados con su estado físico. En particular, si nos referimos a los modelos incluyendo los dos controles de salud (última especificación en ambas tablas), las mujeres obesas mórbidas afirman que su salud es un $21,6 \%$ peor que sus pares en el rango de peso normal y tienen un $9,1 \%$ más de probabilidades de contraer una enfermedad. Por su parte la salud de las obesas cae en un 5,7\% y su probabilidad de estar enferma se eleva a $4,5 \%$ por sobre las mujeres con IMC dentro de lo considerado normal.

Por último estimamos la probabilidad de empleo de los individuos con el objeto de analizar una posible discriminación en esta etapa de la estructura de trabajo ${ }^{12}$. De esta forma, los resultados de (3) muestran que la probabilidad de ser contratadas es un 6,8\% inferior para las mujeres obesas mórbidas y un 2,2\% para las obesas. Luego, al agregar los controles de salud, la primera cifra se reduce a un $6,3 \%$, mientras que la segunda refleja una diferencia negativa de $1,9 \%$ con respecto al rango normal, aunque deja de ser significativa. 


\section{Tabla 8: Empleo}

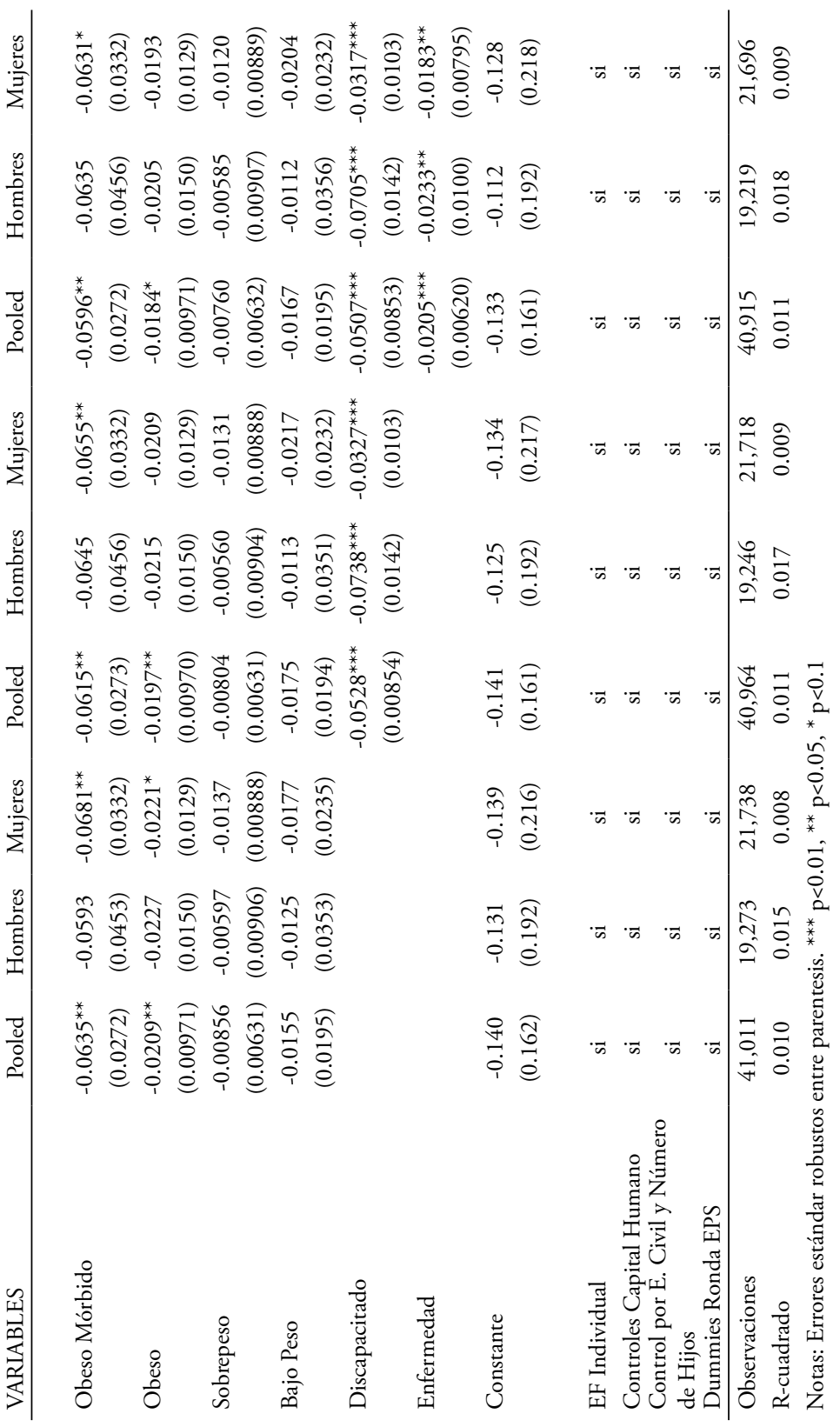


La última tabla de anexos revisa esta relación (3) pero con la medida continua del IMC, encontrando que a medida que aumenta marginalmente la masa corporal de las mujeres, disminuye su probabilidad de encontrar empleo. Luego, podemos adjudicar parte de este efecto a la discriminación, ya sea por parte del empleador o el cliente, pero en la especificación que incluye el cuadrado del IMC también se encuentra un efecto negativo, por lo que esta no linealidad podría perfectamente relacionarse con salud.

Haciendo un repaso de los resultados encontrados, podemos establecer que la obesidad tiene un efecto por si sola en las medidas revisadas, lo que podríamos asociar con discriminación, ya que no poseemos ninguna medida que controle por este factor. $\mathrm{Al} \mathrm{mismo}$ tiempo, se constató un efecto importante sobre la salud de los individuos lo que también estaría afectando indirectamente los resultados laborales. Por tanto, es posible distinguir dos mecanismos a través de los cuales la obesidad podría tener un impacto en el mercado laboral, efecto que se concentra exclusivamente en las mujeres.

\section{Conclusiones}

La idea de realizar este estudio surge por la magnitud del problema de obesidad en Chile, el cual fue expuesto brevemente en la sección de datos para contextualizar la situación a nivel nacional. Esta realidad, sin embargo, no es ajena a varios países de la región y por ende, es importante llevar a cabo una estimación seria y consistente sobre los costos económicos que implica tener una sociedad con altos niveles obesidad, sedentarismo y mal estado de salud, como consecuencia de sus malas costumbres alimenticias. En este sentido, establecer una relación entre diferentes medidas del mercado laboral y la masa corporal de los individuos nos permite tener una idea de la gravedad del asunto, mientras que el enfoque propuesto es capaz de reconocer cuál género es el más perjudicado económicamente por esta condición. Esto último es clave como argumento a la hora de diseñar políticas públicas que históricamente en el país han estado 
basadas en la focalización de recursos hacia los grupos más afectados por algún problema social.

Con todo, a pesar de que las limitaciones econométricas en este tipo de estudios no son menores, y los datos utilizados no alcanzan a tener una gran variación debido al corto período de tiempo entre encuestas (lo cual es de vital importancia para un panel), fuimos capaces de encontrar resultados bastante robustos a las diferentes especificaciones utilizadas, resaltando el $14 \%$ de penalidad en el salario de las mujeres obesas mórbidas, el 7,4\% menos de probabilidad de participación y el 6,3\% menos de probabilidad de empleo, con respecto a sus pares con rango de IMC normal. Por su parte, en participación y probabilidad de empleo, también fue posible distinguir un efecto negativo para las mujeres obesas, aunque de menor magnitud (alrededor del 2\%).

La inclusión de las regresiones de salud fue motivada en primera instancia por la necesidad de demostrar empíricamente la correlación negativa entre la obesidad y salud, a la vez que era importante comprobar si este era uno de los canales a través de los cuales se podrían estar reflejando las perdidas en el mercado del trabajo. Como se vio, las mujeres con un IMC mayor a treinta presentan un peor estado de salud y mayor probabilidad de contraer enfermedades ligadas de una u otra manera a desordenes nutricionales, lo que se condice con los efectos encontrados en los resultados laborales. Así, se cumple el primer objetivo (al menos para las mujeres) y además podemos establecer que la obesidad, a pesar de mostrar un efecto independiente sobre los resultados laborales, correlaciona fuertemente con las medidas de salud revisadas. Por lo tanto, es posible inferir un efecto indirecto a través de este canal.

Finalmente, como dejamos en claro que podría existir más de una explicación para que los obesos sufran pérdidas en el ámbito laboral, resolvimos estimar la probabilidad de empleo en nuestra muestra, ya que es en esta etapa de la estructura laboral (a la hora de contratar o no a alguien) donde se puede asociar mejor una potencial discriminación. Así, encontramos una menor probabilidad de empleo para las mujeres obesas mórbidas, lo que sumado a los 
resultados de las regresiones con la variable IMC continua, nos permite distinguir (además de su efecto directo) dos mecanismos a través de los cuales la obesidad refleja perdidas en el mercado laboral: Salud y discriminación.

\section{Anexos}

Tabla 1. Toda la muestra

\begin{tabular}{|c|c|c|c|c|c|}
\hline 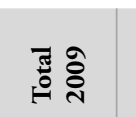 & 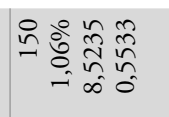 & 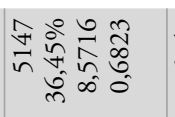 & 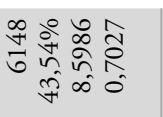 & 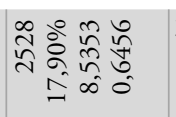 & 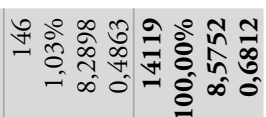 \\
\hline 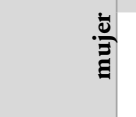 & 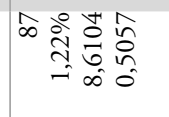 & 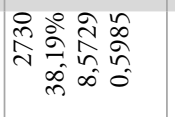 & 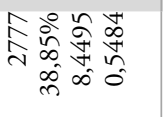 & 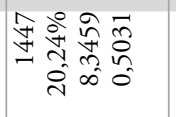 & 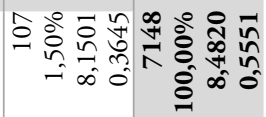 \\
\hline 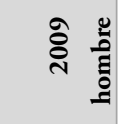 & 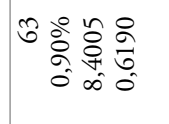 & 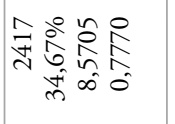 & 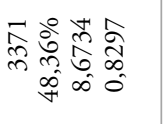 & 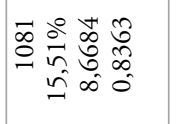 & 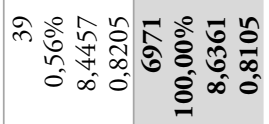 \\
\hline 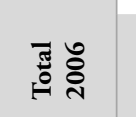 & 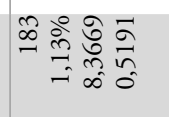 & 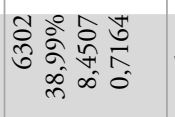 & 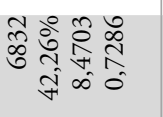 & 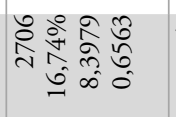 & $\begin{array}{c}\infty \\
\infty \\
0 \\
0\end{array}$ \\
\hline$\frac{\grave{g}}{\bar{z}}$ & 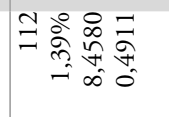 & 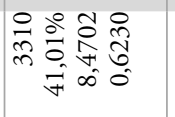 & 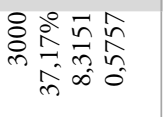 & 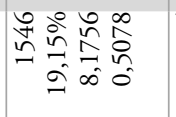 & ए人 \\
\hline ๕ั้ & 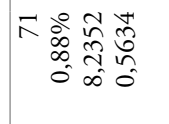 & 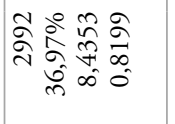 & 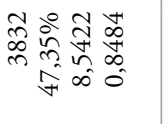 & 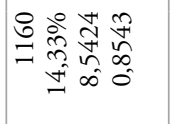 & $F$ \\
\hline 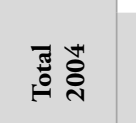 & 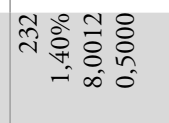 & 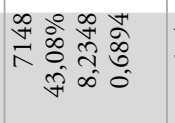 & 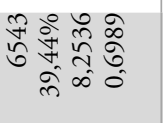 & 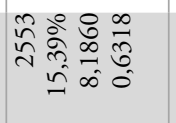 & $\begin{array}{l}\hat{0}_{0} \\
0\end{array}$ \\
\hline$\stackrel{\bar{g}}{\vec{\Xi}}$ & 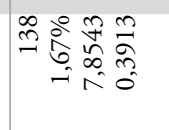 & 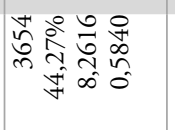 & 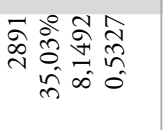 & 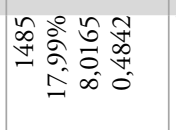 & $\infty$ 요 \\
\hline ثั่ & 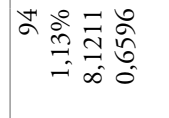 & 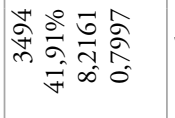 & 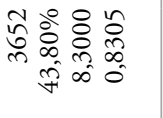 & 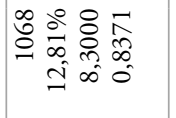 & 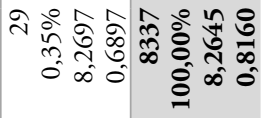 \\
\hline 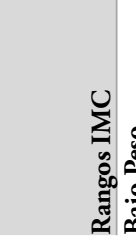 & 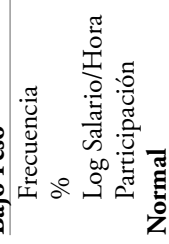 & 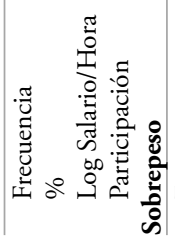 & 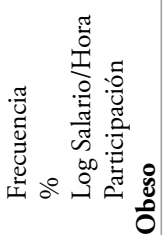 & 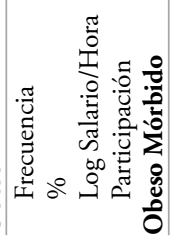 & 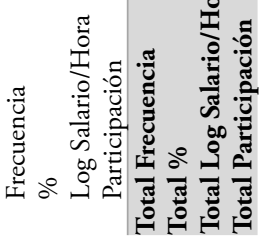 \\
\hline
\end{tabular}


TABla 2. Fuerza laboral (SIN CONTAR TRABAJAdORES POR CUENTA PROPIA NI FFAA).

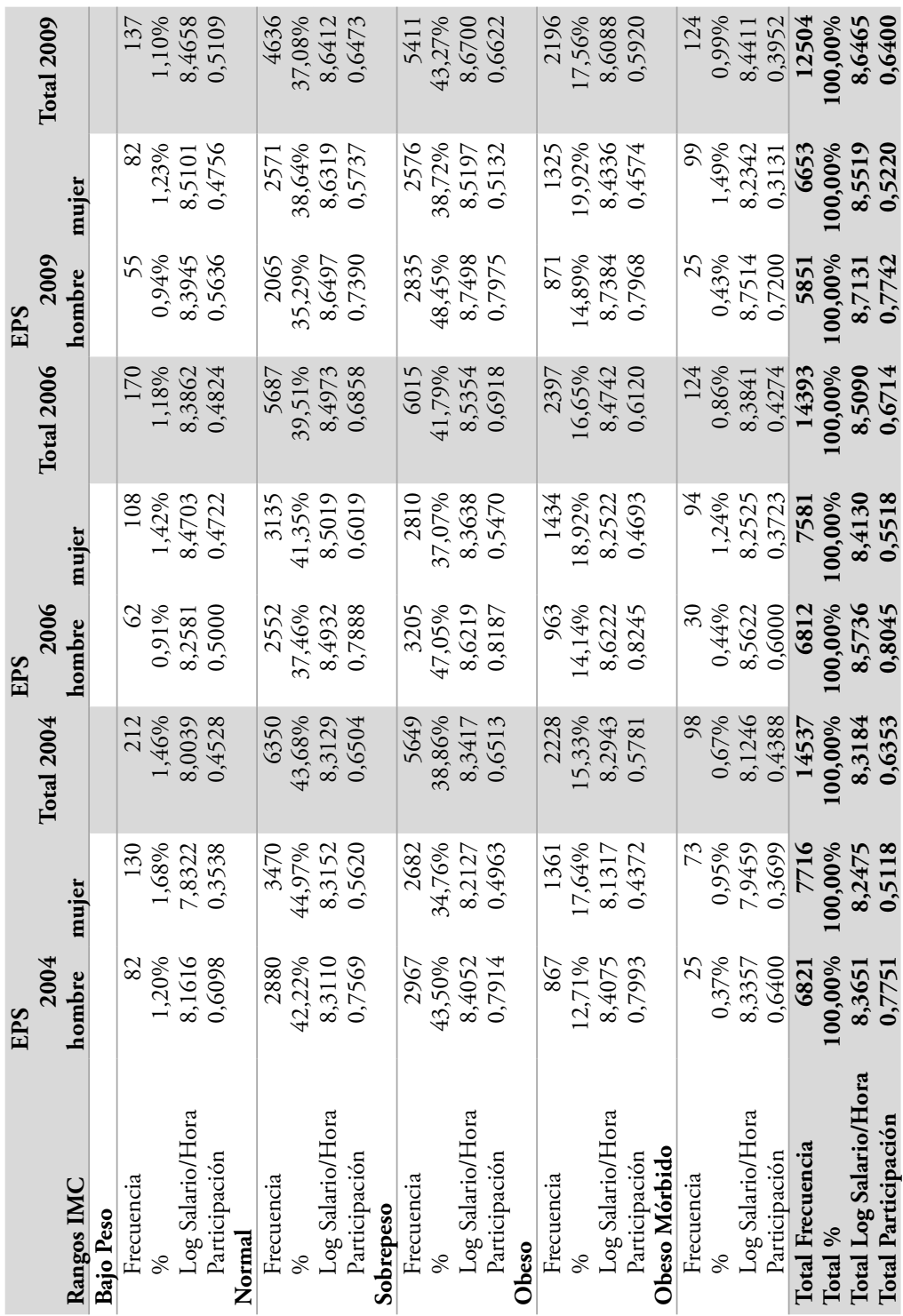


Tabla 3. Salarios IMC

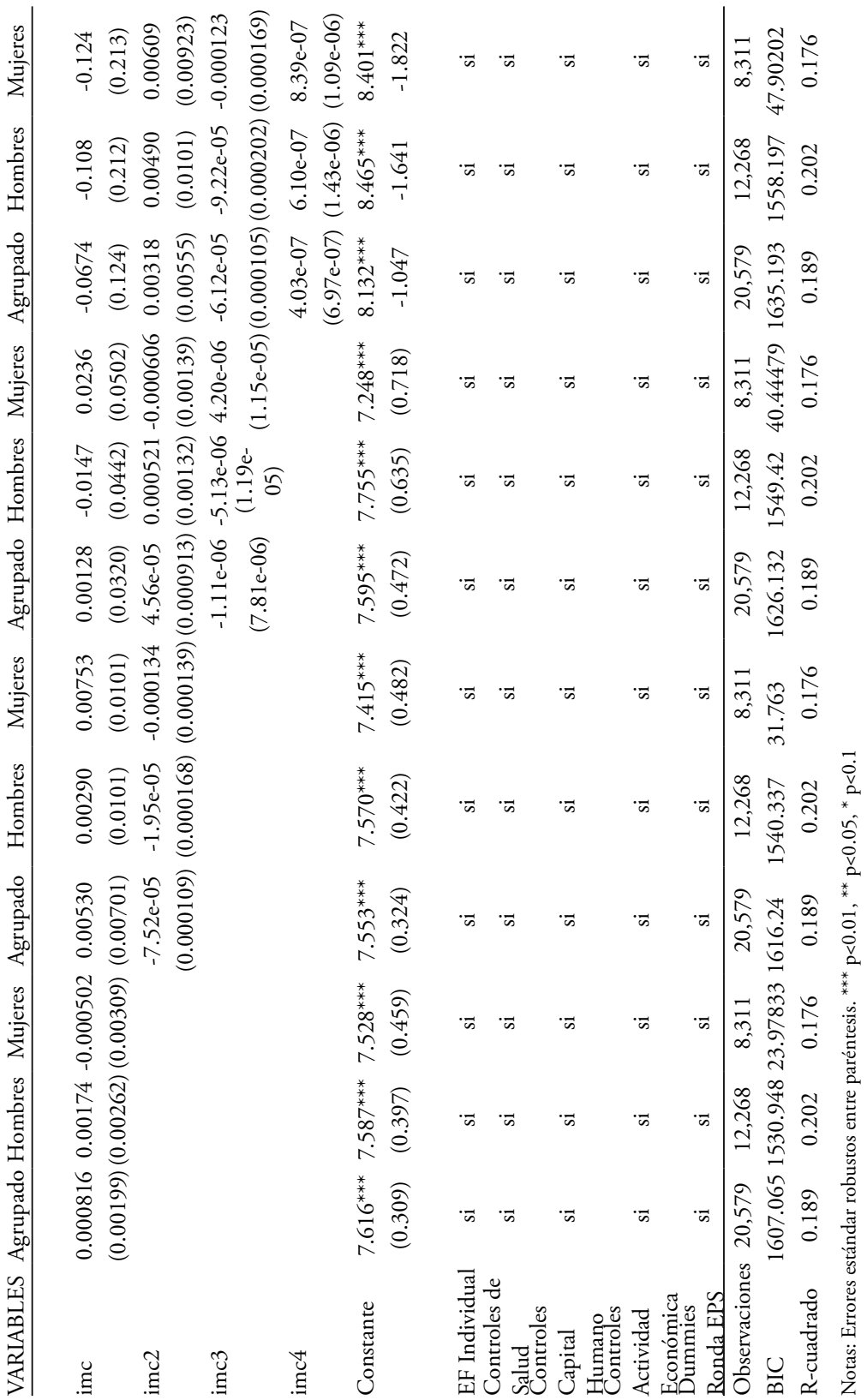




\section{Tabla 4. Participación IMC}

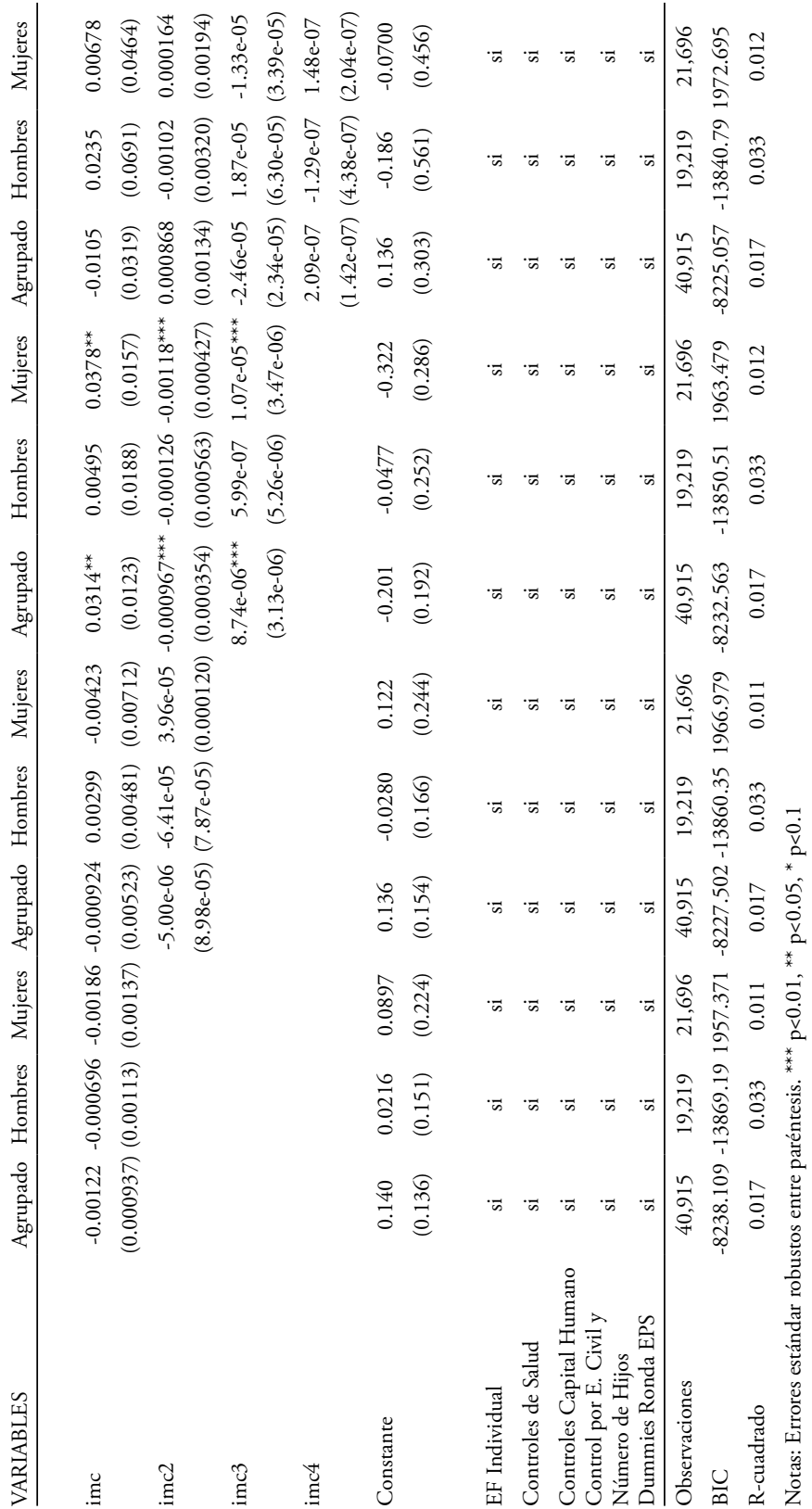


Tabla 5. Empleo IMC

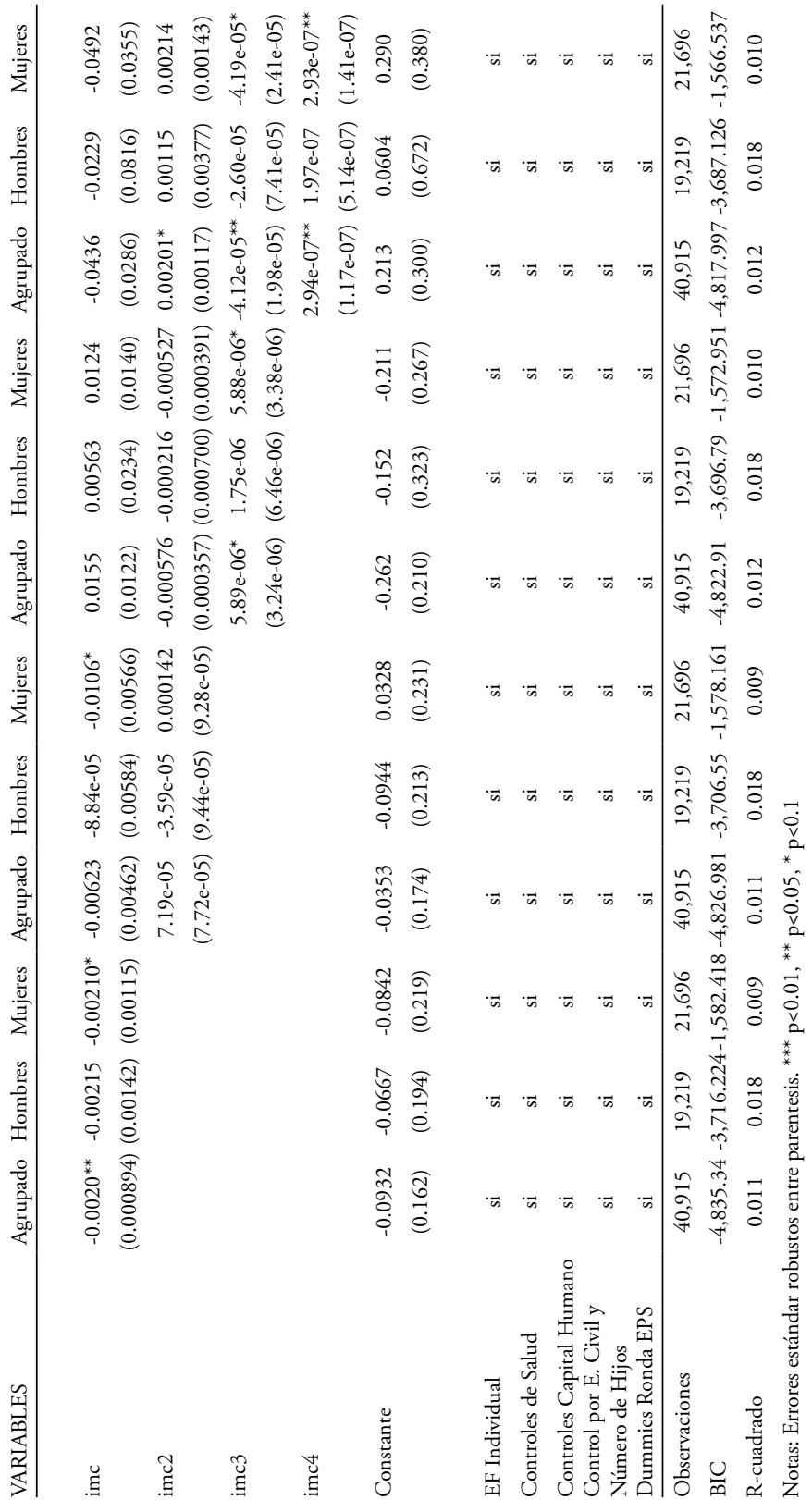


Bibliografía

AVERETT, S. and Korenman, S. (1996). The economic reality of the beauty myth. Journal of Human Resources, 31(2), 304330.

BAUM, C. L. and Ford, W. F. (2004). The wage effects of obesity: A longitudinal study. Health Economics, 13(9), 885-899.

CAWLEY, John. An instrumental variables approach to measuring the effect of body weight on employment disability. Health Services Research. 2000; 35(5):1159-1179. [PubMed: 11130815]

CAWLEY, John. The impact of obesity on wages. Journal of Human Resources. 2004; 39(2):451-74.

CONLEY, Dalton and Glauber, Rebecca. Gender, body mass and economic status. NBER working paper 11343. May, 2005.

COSTA-FONT, Joan and Gil, Joan. Social interactions and the contemporaneous determinants of individuals weight, Applied Economics (2004), vol. 36, pp. 2253- 2263.

D'HOMBRES, B \& Brunello, G. (2005). Does obesity hurt your wages more in Dublin than in Madrid? Evidence from ECHP. IZA Discussion Paper No. 1704, Bonn.

GORTMAKER, Steven; Must, Aviva; Perrin, James; Sobol, Arthur; Dietz, William (1993). Social and Economic Consequences of Overweight in Adolescence and Young Adulthood. New England Journal of Medicine, 1993.

LEIBENSTEIN, Harvey. Economic backwardness and economic growth: Studies in the theory of economic development. New York: Wiles \& Sons, 1957.

LUNDBORG, P; Bolin, K; Höjgård, S. and Lindgren, B. (2007). Obesity and occupational attainment among the 50+ of Europe, in: Bolin, Kristian and Cawley, John (Eds). The Economics of Obesity. Advances in Health Economics and Health Services Research (Vol. 17 pp. 221-54).

MORRIS, S. (2006). Body Mass Index and Occupational Attainment. Journal of Health Economics, 25:347-364.

MURPHY, Kevin M. and Topel, Robert H. Estimation and Inference 
in Two-Step Econometric Models. Journal of Business and Economic Statistics, 3, 4 (1985): 370-379.

NORTON, E. C; Han, E and Stearns, S. C. Do fat workers earn lean paychecks? The University of North Carolina; Chapel Hill: 2008.

NORTON, Edward and Han, Euna (2008). Genetic Information, Obesity and Labor Market Outcomes. Health Economics (Volume 17, Issue 9, pages 1089-1104).

PAGAN, José and Davila, Alberto. Obesity, occupational attainment, and earnings. Social Science Quarterly, 1997.

GARCIA, J. and Quintana-Domeque, C. (2006). Obesity, employment and wages in Europe. Advances in Health Economics and Health Services Research.

SBU (Statens Beredning för Medicinsk Utvärdering). Obesidad problemas e intervenciones. En systematisk översikt. Göteborg: Elanders Digitaltryck AB, 2002.

SARGENT, J. D. and Blanchflower, D. G. (1994). Obesity and stature in adolescence and earnings in young adulthood. Analysis of a British birth cohort. Archives of Pediatrics and Adolescent Medicine, 148, 681-687.

SOBAL, Jeffery (2004). Sociological Analysis of the Stigmatisation of Obesity, in John Germov and Lauren Williams (Editors), A Sociology of Food and Nutrition. The Social Appetite, Oxford, Oxford University Press.

SOUSA, S. (2005). Does size matter? A propensity score approach to the effect of BMI on labour market outcomes. Unpublished manuscript, European University Institute and University of Minho.

STRAUSS, John and Thomas, Duncan. "Health, Nutrition, and Economic Development" Journal of Economic Literature, Vol. 36, No. 2. (Jun., 1998), pp. 766-817.

ZHANG, Lei and Rashad, Inas. Obesity and time preference: The health consequences of discounting the future. Journal of Biosocial Science 40 (1): 97 (2008).

ATALAH, Eduardo (2012), Epidemiología de la Obesidad en Chile. 
Rev. Médica Clínica Las Condes; 23(2) 117-123.

COLAGIURI, Stephen; Lee, Crystal; Colagiuri, Ruth; Magliano, Dianna; Shaw, Jonathan; Zimmet, Paul and Caterson, Ian. The cost of overweight and obesity in Australia. Med J Aust. 2010; 192: 260-4.

COSTA-FONT, Joan and Gil, Joan. Obesity and the incidence of chronic diseases: a seemingly unrelated probit approach. Documents de Treball de la Facultat de Ciencies Economiques I Empresarials, UB. Collecció d'Economia, 2005.

NI MHURCHU C; Rodgers, A; Pan, W. H; Gu, D; Woodward, M. Asia Pacific Cohort Studies Collaboration. Body mass index and cardiovascular disease in the Asia-Pacific region: an overview of 33 cohorts involving 310000 participants. Int J Epidemiol 2004; 33: 751-758.

FIELD, A. E; Coakley, E. H; Must, A; Spadanao, J. L; Laird, N; Dietz, W. H; Rimm, E. and Colditz, G. Impact of overweight on the risk of developing common chronic diseases during a 10-year period. Arch Intern Med 2001; 161:1581-86.

UAUY, R; Albala, C and Kain, J. Obesity trends in Latin America: Transition from under to Overweight. J. Nutr. March 1, 2001 vol. 131 no. 3 893S-899S.

VIO, F; Albala, C. and Kain, J. Nutrition transition in Chile revisited: mid-term evaluation of obesity goals for the period 2000-2010. Public Health Nutr. 2008; 11: 405-12.

NotAs DE PÁGINA:

${ }^{1}$ Véase por ejemplo The cost of overweight and obesity in Australia, Colagiuri et. al (2010). Med J Aust. 2010; 192: 260-4.

${ }^{2}$ Revisar referencias de salud 2,3 y 4.

${ }^{3}$ Esta explicación también sirve para argumentar la elección de modelos de efectos fijos por sobre aleatorios para las ecuaciones de salarios y salud.

${ }^{4}$ Esto se logra utilizando el comando "cluster" en STATA, el cual 
agrupa las observaciones de la misma persona.

${ }^{5}$ Revisar tabla 1 en la sección Datos.

'Participación implica estar trabajando, cesante o buscando trabajo por primera vez.

${ }^{7}$ Excelente $=1 ;$ Muy buena=2; Buena=3; Regular $=4 ;$ Mala=5; Muy mala=6.

${ }^{8}$ En primera instancia (outliers) se eliminan 135 observaciones, equivalentes al $0,29 \%$ de la muestra total. Mientras que los trabajadores por cuenta propia y FFAA que son removidos representan el 11,6\% de la muestra (5.441 observaciones).

${ }^{9}$ Ministerio de Salud. ENS 2003, 2010. Disponible: http://web. minsal.cl/estudios-y-encuestas-de-salud/.

${ }^{10} \mathrm{Cada}$ tabla de resultados tendrá bien especificado los controles utilizados en la estimación.

${ }^{11}$ Asma y/o efisema, diabetes, depresión, hipertensión o problemas cardíacos.

${ }^{12}$ Considerando una primera etapa de decisión de participación, una segunda de encontrar (o no) empleo y una tercera de optar por un cierto monto de salario.

Fecha de Recepción del Artículo: 12 de enero de 2018 Fecha de Aceptación: 25 de junio de 2018 